\title{
A literacia para a saúde no setting escolar
}

\author{
Kéllen Campos Castro Moreira ${ }^{1}$, Rosane Aparecida de Sousa Martins ${ }^{2}$, Luís Saboga-Nunes ${ }^{3}$
}

\begin{abstract}
Resumo
O trabalho do tema saúde, na escola, exige uma abordagem centrada na atenção integral e concepção holística, pautada no diálogo e na construção coletiva para que, por meio da educação para a saúde, seja possível capacitar as crianças para acessar, compreender, avaliar, gerir e aplicar os conhecimentos, a partir de comportamentos favoráveis à saúde, pelo aumento do nível de literacia para a saúde. Ressalta-se a relevância de ampliar o debate acerca do documento que norteia a atuação docente nas escolas, Parâmetros Curriculares Nacionais sobre Ciências Naturais que, apesar de apresentar uma proposta sobre a temática de saúde, define assuntos direcionados aos temas que focam em doenças e tratamentos.
\end{abstract}

\section{Palavras-chave}

Literacia. Saúde. Escola.

\footnotetext{
${ }^{1}$ Mestra em Psicologia pela Universidade Federal de Uberlândia, Minas Gerais, Brasil; membro do Grupo de Estudos e Pesquisa Promoção em Comunicação, Educação e Literacia para a Saúde no Brasil (ProLiSaBr) da Universidade Federal do Triângulo Mineiro, Minas Gerais, Brasil; enfermeira assistencial na Prefeitura Municipal de Uberaba, Minas Gerais, Brasil. E-mail: kellen_camposcastro@ yahoo.com.br.

${ }^{2}$ Doutora em Serviço Social pela Universidade Estadual Paulista Júlio de Mesquita Filho, São Paulo, Brasil; professora titular da Universidade Federal do Triângulo Mineiro, Minas Gerais, Brasil; coordenadora do Grupo de Estudos e Pesquisa Promoção em Comunicação, Educação e Literacia para a Saúde no Brasil (ProLiSaBr/UFTM); membro do Grupo de Pesquisa Ciclos de Vida, Família e Saúde no Contexto Social (FACS/UFTM); colaboradora no Programa de Residência Integrada e multiprofissional em Saúde (RIMS/UFTM). E-mail: drarosane.martins@gmail.com.

${ }^{3}$ Doutor em Saúde Pública pela Universidade Nova de Lisboa, Portugal; professor na Escola Nacional de Saúde Pública da Universidade Nova de Lisboa, Portugal; professor associado na Universidade de Educação de Freiburg, Alemanha. E-mail: saboga.nunes@gmail.com.
} 


\title{
Health literacy in the school setting
}

Kéllen Campos Castro Moreira ${ }^{4}$, Rosane Aparecida de Sousa Martinss ${ }^{5}$ Luís Saboga-Nunes ${ }^{6}$

\begin{abstract}
The work regarding health, in the school requires an approach centered on integral attention and holistic conception, based on dialogue and collective construction so that through health education it is possible to enable children to access, understand, evaluate, manage and apply knowledge, through health-promoting behaviors, by raising the level of the health literacy. It is important to expand the debate about the document that guides the teaching performance in schools, Parâmetros Curriculares Nacionais sobre Ciências Naturais which, despite presenting a proposal on health issues, defines subjects around themes that focus on diseases and treatments.
\end{abstract}

\section{Keywords}

Literacy. Health. School.

\footnotetext{
${ }^{4}$ Master in Psychology, Federal University of Uberlândia, State of Minas Gerais, Brazil; member of the Group of Studies and Research Promotion on Communication, Education and Literacy for Health in Brazil (ProLiSaBr) of the Federal University of Triângulo Mineiro, State of Minas Gerais, Brazil; clinical nurse in the Uberaba City Hall, State of Minas Gerais, Brazil. E-mail: kellen_camposcastro@ yahoo.com.br.

${ }^{5} \mathrm{PhD}$ in Social Work, Paulista State University Júlio de Mesquita Filho, State of São Paulo, Brazil; full professor at the Federal University of Triângulo Mineiro, State of Minas Gerais, Brazil; coordinator of the Study Group and Research Promotion on Communication, Education and Literacy for Health in Brazil (ProLiSaBr/UFTM); member of the Research Group Life Cycles, Family and Health in the Social Context (FACS/UFTM); collaborator in the Multiprofessional Integrated Residency Program in Health (RIMS/UFTM). E-mail: drarosane.martins@gmail.com.

${ }^{6} \mathrm{PhD}$ in Public Health, New University of Lisbon, Portugal; professor at the National School of Public Health, New University of Lisbon, Portugal; associate professor at the Freiburg University of Education, Germany. Email: saboga.nunes@gmail.com.
} 


\section{Introdução}

\section{Escola: um setting primordial para buscar as origens da saúde}

A infância é apontada como fase de desenvolvimento propícia para promover comportamentos de saúde através da educação (BRASIL, 2002; CARVALHO; JOURDAN, 2014). Uma parte significativa da infância é passada na escola, sendo setting, um espaço propício à promoção da saúde. Consequentemente, ações intersetoriais estabelecendo parcerias entre os setores da saúde e da educação, como o Programa Saúde Escola (BRASIL, 2009), revelam-se muito promissoras. Tentativas governamentais visando implementar políticas e programas para a promoção da saúde mostram-se diversas vezes reprodutoras do modelo patogênico da saúde, ou seja, os profissionais, ao tentar abordar sobre assuntos relativos à saúde acabam por desenvolver atividades com enfoque na doença ou restritas ao seu tratamento. Exemplos disso são os temas que tradicionalmente fazem parte dos projetos de educação para a saúde nas escolas, como piolho, Infecções Sexualmente Transmissíveis (IST), verminoses, dentre outros. Por outro lado, assuntos como cultura de paz, habitação, família, educação, espiritualidade, lazer, determinantes e condicionantes de saúde, e aspectos reveladores da promoção da saúde (como reposição hídrica, utilização do sol e síntese da vitamina D) estão fora dos programas (MOREIRA, 2016).

No Brasil, em alguns casos, há parceria com Unidades Básicas de Saúde através de seus profissionais que realizam, esporadicamente, atividades de educação para a saúde com as crianças. No entanto, os conhecimentos e as competências referentes à saúde são abordados como uma pequena parte de conteúdo, dentro de uma disciplina denominada de Ciências Naturais ou Biologia Humana, cujo norte ocorre por meio do documento intitulado Parâmetros Curriculares Nacionais (PCN) sobre Ciências Naturais. Dele até o terceiro e quarto ciclos do ensino fundamental, constam como eixos temáticos: Terra e Universo, Vida e Ambiente, Ser Humano e Saúde, Tecnologia e Sociedade, Temas Transversais e Ciências Naturais. Em relação ao Ser Humano e Saúde o foco é o desenvolvimento humano pelos ciclos da vida e o funcionamento do corpo. O tema transversal Saúde "aborda as relações entre os problemas de saúde e fatores econômicos, políticos, sociais e históricos" (BRASIL, 1998, p. 46), ou seja, direciona-se para a relação indissociável entre saúde e fatores sóciohistórico-econômicos e culturais. É possível identificar na citação acima que, apesar de trazer a palavra saúde no eixo transversal, os PCN sobre Ciências Naturais ratificam uma visão focada na doença, ou seja, há predomínio da visão patogênica quando se propõe a abordar 
questões relacionadas com a saúde, em detrimento da visão salutogênica (SABOGA-NUNES, 2019) (sobre saúde, suas origens, seus determinantes e condicionantes):

Em Ciências Naturais, apresentar a saúde como um estado de equilíbrio dinâmico do corpo e um bem da coletividade é uma meta que não é simples e que precisa ser reiterada em diferentes momentos, por meio de abordagens diversificadas. Os nomes de doenças, seus agentes e sintomas são conteúdos desenvolvidos em temas de trabalho significativos para os estudantes, como, por exemplo, a investigação dos meios de combate à dengue, mas é de pouca valia sua apresentação isolada de contexto. (BRASIL, 1998, p. 46).

Em um esforço para superar a perspectiva biomédica de foco patogênico, destaca-se em outro trecho a tentativa de abordar o tema Saúde numa perspectiva de promoção da saúde nos conteúdos escolares: “oferecem perspectivas sociais e ambientais que ampliam a abordagem tradicional de programas de saúde nos currículos de Ciências, cuja tônica tem sido o estudo das doenças e não o desenvolvimento da saúde" (BRASIL, 1998, p. 51). Desse modo, apesar do reconhecimento pelo próprio órgão governamental da importância em abordar temas de saúde na perspectiva salutogênica, na prática reconhece-se uma dificuldade na superação do Modelo Biomédico, construído historicamente na sociedade ocidental, cujo foco de atenção à saúde ainda está centrado na doença e no seu tratamento. Essa perspectiva está ancorada no paradigma Flexneriano, cujo foco baseia-se na concepção mecanicista do processo saúdedoença, reducionista, e fragmentado em disciplina e especialidades.

\section{Educação para promover saúde ou prevenir doenças?}

A discussão sobre a abordagem do tema saúde nas escolas tem sido reconhecida como extremamente importantes, segundo a Organização Mundial de Saúde, e tem sido tratada de forma diversa em cada país (CARVALHO; JOURDAN, 2014). No Brasil, muitas vezes a educação para a saúde não acontece na atuação profissional devido à perda de verdadeiros conceitos-chave. Esse fato pode ser exemplificado no caráter informativo de atividades denominadas educativas; ou de considerar aferição de pressão arterial como medida de promoção da saúde ao invés de preventiva de doenças/complicações; ou desconsiderar que para uma comunicação efetiva deve-se ir além da informação (mensagem) e desenvolver a literacia para a saúde (LS); ou de apresentar um conceito patogênico do processo saúdedoença. Preconiza-se por isso, que a educação para a saúde não se subjugue à verticalização do saber científico com discursos taxativos e impositivos, mas que haja diálogo, interação 
social, relação entre os indivíduos e o contexto sociocultural (ALVES, 2017; BRASIL, 2012; CARVALHO; JOURDAN, 2014; VARGAS; PINTO; MARINHO, 2019; MOREIRA, 2016).

Para ocorrer a comunicação, deve-se envolver informações em uma troca recíproca entre locutor e receptor, a certificação da compreensão da mensagem pelo outro, informações que estejam mescladas aos aspectos culturais, sociais e de experiências individuais (VARGAS; PINTO; MARINHO, 2019; MOREIRA, 2016). É imprescindível, no processo educativo, perceber a saúde como compreensão mais ampla, muito além de ausência de doenças ou de um bem-estar momentâneo. Nesse âmbito, pode-se compreender a educação para a saúde como uma estratégia de promoção da saúde (BRASIL, 2010). Dessa forma, a promoção da saúde tem o potencial para fomentar o processo de capacitação das pessoas para o cuidado de si e de sua comunidade em termos de saúde, qualidade de vida e estilo de vida saudáveis (WILBERG; SABOGA-NUNES; STOCK, 2019), e a LS como resultado da promoção da saúde, por meio da ampliação do controle sobre a saúde e seus determinantes (CARVALHO; JOURDAN, 2014). Tal assertiva ratifica a relevância da abordagem da temática de LS como estratégia para fortalecimento de ações com enfoque na promoção da saúde no contexto escolar.

\section{Compreensões sobre literacia para a saúde}

A LS pode ser conceituada como um campo de investigação e atuação das ciências sociais que visa a combater as disparidades sociais em saúde (SABOGA-NUNES et al., 2014). Consiste numa visão complexa e holística da vida em sociedade, e envolve um processo continuo de aprendizagem e capacitação da pessoa para acessar, compreender, avaliar, gerir e aplicar os conhecimentos de forma favorável a aumentar o controle sobre a saúde e melhorála, em nível individual e coletivo. É relevante para promover saúde, melhorar o nível de qualidade de vida e auxiliar na capacidade e motivação para escolhas mais saudáveis (SABOGA-NUNES et al., 2014; SABOGA-NUNES et al., 2019; VARGAS; PINTO; MARINHO, 2019).

O nível de LS em que um indivíduo se encontra pode ser avaliado por instrumentos que aferem competências e habilidades em saúde quanto à cura e cuidados, prevenção de doença e promoção da saúde, bem como quanto às suas dimensões (acesso à informação, compreensão 
da informação, avaliação, capacidade de gerir e aplicar os conhecimentos para aumentar o controle sobre a saúde) (MADEIRA, 2016; SABOGA-NUNES et al., 2014; SILVA, 2017).

Estudos apontam que baixos níveis de LS indicam fracos resultados em saúde, por exemplo, em pacientes crônicos que desenvolvem menor capacidade de autocuidado e maiores usos de serviços de saúde (CARVALHO; JOURDAN, 2014; RAN et al., 2018; SABOGA-NUNES et $a l .$, 2014). Em relação à LS com estudantes, pode-se exemplificar um estudo recente que concluiu, por diferentes escalas, que altos níveis de LS estiveram associados com maiores índices de qualidade de vida. $\mathrm{O}$ resultado dessa investigação apontou para a urgência em haver esforços de saúde pública para melhorar a promoção e educação para a saúde, especialmente em estudantes do nível secundário com baixa LS (RAN et al., 2018). Percebese que a LS não consiste apenas em medir níveis da população quanto ao processo saúdedoença, mas é resultado da promoção da saúde. Portanto, a LS possibilita o aumento, pelas pessoas, do controle sobre a sua saúde, e consequentemente melhorias, não se limitando ao gerenciamento apenas individual, mas também dos determinantes de saúde ao nível pessoal, social e ambiental (CARVALHO; JOURDAN, 2014).

\section{Considerações finais}

A perspectiva de se trabalhar o tema de saúde na escola exige uma abordagem na qual a promoção da saúde esteja no mesmo patamar que prevenção, reabilitação e o tratamento das doenças. Além disso, é mister que os programas com ênfase na saúde nas escolas tenham como premissa a concepção holística do ser humano e uma abordagem centrada na atenção integral, visão generalista, pautada no diálogo e na construção coletiva, e com temáticas que foquem na saúde (salutogênese).

Portanto, ressalta-se a relevância de ampliar o debate acerca dos PCN Ciências Naturais, da interface entre educação e saúde na escola, de questionar a formação dos professores brasileiros e dos profissionais de saúde. Assim talvez, futuramente, a educação para a saúde se torne uma disciplina composta por equipes multiprofissionais nas escolas, e possibilite capacitar as crianças para acessar, compreender, avaliar, gerir e aplicar os conhecimentos, através de comportamentos favoráveis à saúde, pelo aumento do nível de LS. 


\section{Referências}

ALVES, M. R. Educação popular em saúde: um referencial de autonomia e transformação. 2017. 91 f. Dissertação (Mestrado em Ensino em Saúde) - Universidade Federal dos Vales do Jequitinhonha e Mucuri, Diamantina, 2017.

BRASIL. Ministério da Educação. Secretaria de Educação Fundamental. Parâmetros Curriculares Nacionais: Ciências Naturais. Brasília: MEC/SEF, 1998.

BRASIL. Ministério da Saúde. A promoção da saúde no contexto escolar: informes técnicos institucionais. Brasília: MS, 2002.

BRASIL. Ministério da Saúde. Cadernos de Atenção Básica 24: Programa Saúde na Escola (PSE). Brasília: MS, 2009.

BRASIL. Ministério da Saúde. Cadernos de Atenção Básica 33: Saúde da criança: crescimento e desenvolvimento. Brasília: MS, 2012.

BRASIL. Ministério da Saúde. Política Nacional de Promoção da Saúde. 3. ed. Brasília: MS, 2010.

CARVALHO, G. S.; JOURDAN, D. Literacia em saúde na escola: a importância dos contextos sociais. In: JÚNIOR, C. A. O. M., JÚNIOR, Á. L., CORAZZA, M. J. (org.). Ensino de ciências: múltiplas perspectivas, diferentes olhares. Curitiba: CRV, 2014. p. 99122.

MADEIRA, T. F. M. Literacia para a saúde: o perfil de um conceito em construção. 2016. 64 f. Dissertação (Medicina) - Universidade da Beira Interior, Covilhã, 2016.

MOREIRA, K. C. C. Intervenção mediacional e promoção da saúde: um estudo com crianças pré-escolares. 2016. 166 f. Dissertação (Psicologia) - Universidade Federal de Uberlândia, Uberlândia, 2016.

RAN, M. et al. The association between quality of life (QOL) and health literacy among junior middle school students: a cross-sectional study. BMC Public Health, v. 1.183, n. 18, 2018. Doi: 10.1186/s12889-018-6082-5.

SABOGA-NUNES, L.; BITTLINGMAYER, U.; OKAN, O. Salutogenesis and health literacy: the health promotion simplex! In: OKAN, O. et al. (org.) International handbook of health literacy: research, practice and policy across the lifes-pan. Bristol: The Policy Press, University of Bristol, England, 2019.

SABOGA-NUNES, L. et al. Hermenêutica da literacia em saúde e sua avaliação em Portugal (HLS-U-PT). In: VIII CONGRESSO PORTUGUÊS DE SOCIOLOGIA, 2014, Portugal. Disponível em:

http://historico.aps.pt/viii_congresso/VIII_ACTAS/VIII_COM0526.pdf.

SABOGA-NUNES, L. et al. Literacia para a saúde: origens e implicação do conceito. In: O papel da literacia para a saúde e educação para a saúde na promoção da saúde. Curitiba: CRV, 2019. 
SILVA, T. L. Contribuições da Literacia em Saúde (Health Literacy) para o aprimoramento das ações de educação em saúde na Atenção Básica. 2017. 265 f. Tese (Doutorado em Ciências) - Escola Nacional de Saúde Pública Sergio Arouca, Fundação Oswaldo Cruz, Rio de Janeiro, 2017.

VARGAS, J. R.; PINTO, M.; MARINHO, S. Desafios da comunicação na prática da literacia em saúde. In: VI JORNADAS DOUTORAIS, COMUNICAÇÃO \& ESTUDOS CULTURAIS, 2019, Braga: CECS. Disponível em:

http://repositorium.sdum.uminho.pt/handle/1822/60251. Acesso em: 12 jun. 2019.

LBERG, A.;, SABOGA-NUNES, L.;, STOCK, C. Are we there yet? Use of the Ottawa charter action areas in the perspective of European health promotion professionals. Journal of Public Health: from theory to practice. Doi: 10.1007/s10389-019-01108-xl.

Submetido em 16 de julho de 2019.

Aprovado em 20 de agosto de 2019. 